\title{
Guidelines for UTI and antenatal hydronephrosis should be gender specific
}

Paediatric patients could benefit from gender-specific guidelines for the prevention of recurrent UTI.

Cystoureterography (VCUG) to investigate UTI is associated with potential risk factors for future infections, and febrile UTI can cause long-term renal damage. At-risk children must undergo further procedures to identify vesicoureteral reflux (VUR) and receive antibiotic prophylaxis. As up to onethird of children with UTI are found to have had VUR, it is thought that VUR predisposes to recurrent infections, and, subsequently, to renal scarring.

Moore et al. evaluated the risk of future febrile UTI by comparing children who had VCUG for a first febrile UTI and newborns who underwent VCUG for antenatal hydronephrosis (ANH). The groups differed in gender distribution, with a female predominance in the UTI group and male predominance for $\mathrm{ANH}$.
Children who underwent VCUG after primary UTI had significantly more consequent UTIs compared with children who had ANH; however, UTI risk did not differ between children with and without VUR. Overall, females had more infections than males, suggesting that female gender was the main risk factor for recurrent febrile UTI. Furthermore, they were also at higher risk of febrile UTI than circumcised males at the age of 0-24 months, regardless of the independent variables of age, presence of VUR, VUR degree, ANH or a previous UTI and study group, suggesting that clinical guidelines for UTI and ANH management would be more beneficial if they addressed male and female patients separately.

Maria Christodoulou

Original article Moore, S. S. et al. Guidelines for urinary tract infections and antenatal hydronephrosis should be gender specific. Acta Paediatr. doi:10.1111/apa.13129 